\title{
Asymptomatic bacteriuria and associated host factors in Diabetic patients with special reference to UTI Chromagar
}

\author{
Divyashree $\mathbf{K}^{1}$, Yadhav $\mathbf{K}^{2}$ \\ ${ }^{1}$ Divyashree K, MBBS Student, ${ }^{2}$ Dr. Kala Yadhav M L, Professor of Microbiology. Both are affiliated with Bangalore \\ Medical College and Research Institute, Fort, KR Road, Bengaluru, Karnataka 560002, India
}

Address for Correspondence: Dr. Kala Yadhav M L, Email ID: kalayadv@rediffmail.com

\begin{abstract}
Introduction: To study the prevalence of asymptomatic bacteriuria (ASB) in diabetics compared to non-diabetics and to determine the associated host factors responsible for ASB in diabetics. Materials and Methods: 250 diabetics and 100 controls were enrolled in the study and were screened for ASB using semi-quantitative culture methods and UTIchrom agar. The samples were screened for glucosuria, ketonuria and pyuria. Results: ASB was more common in diabetics compared to non-diabetics $(12.8 \% \mathrm{v} / \mathrm{s} 6 \%)$ and the increase was more in female diabetics when compared to female nondiabetics $(16 \% \mathrm{v} / \mathrm{s} 6.15 \%)$ whereas male counterparts did not show much difference from the non-diabetics $(6.09 \% \mathrm{v} / \mathrm{s}$ $5.71 \%$ ). There was significant influence of age, duration of diabetes, glucosuria, ketonuria, proteinuria and pyuria on the incidence of ASB in diabetics thus proving them as possible risk factors for development of ASB. E.coli was the most common organism found in both diabetic as well as non diabetic cases of ASB (40.6\% and 57.14\% respectively). The next frequent organisms were Klebsiella and Staphylococcus. The sensitivity pattern was similar in both the population with ASB. Culture on UTI CHROMagar yielded quicker results when compared to standard culture methods. Conclusion: ASB is almost 3 times more common in female diabetics compared to female non-diabetics whereas there is not much difference among the male counterparts. The risk factors for ASB in diabetics included age, longer duration of diabetes, poor glycaemic control and renal dysfunction indicated by proteinuria. Culture on UTI CHROMagar is an easy and faster means of identification of organisms.
\end{abstract}

Keywords: Asymptomatic bacteriuria, Diabetics, Escherichia Coli, pyuria, UTI CHROMagar.

\section{Introduction}

Nearly 61.3 million Indians in their most productive years - (20 to 79 years) - are diabetic, a disease that exposes them to heart attack, stroke, amputations, ner.ve damage, blindness and kidney disease [1]. Diabetes and urologic diseases are very common health problems that markedly increase in prevalence and incidence with advancing age. Severe complications of urinary tract infections occur virtually only in patients with diabetes [2].

Patients with diabetes mellitus have a higher prevalence of asymptomatic bacteriuria (ASB) and incidence of urinary tract infections (UTIs) compared with patients without diabetes mellitus. In fact presence of ASB in patients with Type 2 diabetes acts as a predisposing

Manuscript received: $10^{\text {th }}$ Sept 2015

Reviewed: $24^{\text {th }}$ Sept 2015

Author Corrected: $4^{\text {th }}$ Oct 2015

Accepted for Publication: $16^{\text {th }}$ Oct 2015 factor for subsequent development of a symptomatic UTI [3]. Complications of UTIs such as emphysematous cystitis, pyelonephritis and renal

papillary necrosis occur more commonly in subjects with Type 2 diabetes mellitus [4]. Several risk factors for asymptomatic bacteriuria in diabetics have been suggested (age, disease duration, quality of metabolic control of disease and status of diabetic complications) $[4,5]$.

The frequency of asymptomatic bacteriuria in patients with diabetes mellitus is $9-27 \%$ in females and $0.7-1 \%$ in males [6]. The increased frequency is probably secondary to autonomic neuropathy of the bladder. The static pool of urine due to bladder dysfunction and poor contractility serves as a favourable medium for bacterial growth. Even hyperglycemic urine promotes rapid 
bacterial growth and colonization. Diabetic patients with asymptomatic bacteriuria are more likely to have albuminuria and symptomatic UTIs [7].

Escherchia coli are the most common organism and are most likely to occur in healthy persons. A variety of organisms may be found including Enterobacteriaceae, Klebsiella pneumoniae, Proteus species, Enterococcus species and group B Streptococcus [8]. In men, Enterococcus species and gram-negative bacilli are common.

UTI CHROM agar medium is a chromogenic medium which is known to be a better medium for easy, accurate and early identification of organisms when compared to standard culture medium and is found to be less time-consuming $[9,10]$.

In India, report on ASB in diabetics is scarce and its impact poorly documented. Consequently we decided to study on the frequency of asymptomatic bacteriura in clinically diagnosed patients with diabetes in comparison to non-diabetics and to verify whether there is association between bacteriuria and following suggested risk factors in diabetics - age, sex, duration of diabetes, metabolic control and proteinuria. Also the uropathogens responsible for bacteriuria and their antimicrobial susceptibility was evaluated.

\section{Materials and Methods}

This is a comparative study conducted in the Department of Microbiology over a period of 6 months. Two hundred and fifty patients aged 30-80 years diagnosed with type 2 diabetes mellitus attending outpatient departments for routine check-ups and 100 age related controls were included in the study.

Exclusion criteria included history of UTI symptoms like dysuria, frequency and urgency, patients on urinary catheters or having renal ailments, pregnant women and patients who received antibiotics for the past 2 weeks. Approval to conduct the study was obtained from the ethical committee of the institution. The procedure and purpose of collecting samples for the study was explained to each subject in their own language, highlighting that their personal details and other specificities would not be revealed and that they would not incur any difficulties from participating in the study. Each subject was asked to sign an informed consent giving their permission to participate in the study.
The study group was interviewed and details on demographics (name, age, gender and address) and medical variables (diabetes type, duration and therapy, presence of diabetes related systemic diseases) were recorded in the proforma.

Clean-voided, mid-stream urine samples was collected in wide mouth sterile containers from both case and control groups. The samples were then transported to the microbiology laboratory for immediate processing (within 2 hours). The urine samples were processed according to the standard guidelines [11], culture put up first to prevent contamination and then used to test for urine sugar, ketones, albumin and wet mount examination.

Routine urine samples were cultured on blood agar and MacConkey agar by standard loop method for semiquantitative culture [12]. The inoculated media was incubated aerobically at $37^{\circ} \mathrm{C}$ for $24 \mathrm{hrs}$. A diagnosis of asymptomatic bacteriuria in diabetics as well as non diabetics was made as per the 2005 IDSA guidelines [13]. In those cases with significant bacteriuria, the uropathogens were identified by biochemical reactions using standard methods [11]. Isolated strains were tested for antibiotic susceptibility testing by KirbyBauer's disc diffusion method following Clinical Laboratory Standards Institute (CLSI) guidelines [14].

The urine samples were also simultaneously inoculated on UTICHROMagar while inoculating on standard medium and incubated at $37^{\circ} \mathrm{c}$ for $24 \mathrm{hrs}$. The number and identification of different organisms grown on the UTICHROMagar medium were primarily assessed by colony colour and morphology as mentioned by the manufacturer.

Pyuria indicates inflammatory response in the urinary tract $[11,15]$ so the urine sample is examined by wet mount to detect the presence of pus cells. Glucosuria, ketonuria and proteinuria were evaluated using dip stick method.

Data Analysis: The collected data was statistically analyzed by using chi-square test $(\times 2)$ and $\mathrm{P}$ value determined. T-values were calculated by student's ' $\mathrm{T}$ ' test formula for means \pm standard deviations of ages. $\mathrm{P}$ value of $<0.05$ was considered significant. The data collected was interpreted in tables. 


\section{Results}

Of the 250 diabetic cases enrolled in the study, 82 were males and 168 were females. The rate of ASB was $6 \%$ (5 of 82 ) among male diabetics and 16\% (27 of 168) among female diabetics. There were 100 non-diabetic controls included in the study among which 2 out of 35 males and 4 out of 65 females had ASB accounting for $5.71 \%$ and $6.15 \%$ of incidence of ASB respectively.

It was found that the incidence of ASB was greater in diabetics $(12.8 \%)$ compared to non diabetics $(6 \%)$ [P value < 0.05]. Also among diabetic cases, female diabetics (16\%) had a higher frequency of ASB compared to male diabetics $(6.09 \%)$. There was almost 3 times increased frequency of ASB in female diabetics (16\%) as compared to female nondiabetics $(6.15 \%)$ [P value $=0.02]$ whereas there was only $0.38 \%$ increase in male diabetics $(6.09 \%)$ compared to male non-diabetics $(5.71 \%)$ which is not significant $[\mathrm{P}$ value $=0.48]$.

Table 1: Distribution of ASB with respect to age in diabetic cases

\begin{tabular}{|l|l|l|l|}
\hline Age of the patient & Sample Size & Asb Positives & Percentage \\
\hline $30-40 \mathrm{yrs}$ & 26 & 2 & $7.7 \%$ \\
\hline $40-50 \mathrm{yrs}$ & 68 & 9 & $13.2 \%$ \\
\hline $50-60 \mathrm{yrs}$ & 73 & 8 & $10.9 \%$ \\
\hline $60-70 \mathrm{yrs}$ & 66 & 9 & $13.6 \%$ \\
\hline $70-80 \mathrm{yrs}$ & 17 & 4 & $23.5 \%$ \\
\hline
\end{tabular}

Table 2: Distribution of ASB with respect to duration of diabetes

\begin{tabular}{|l|l|l|l|}
\hline Duration of diabetes & Sample size & Asb Positives & Percentage \\
\hline$<5$ yrs & 122 & 10 & $8.19 \%$ \\
\hline $6-10 \mathrm{yrs}$ & 84 & 14 & $16.67 \%$ \\
\hline $11-15 \mathrm{yrs}$ & 29 & 4 & $13.8 \%$ \\
\hline $16-20 \mathrm{yrs}$ & 12 & 4 & $33.33 \%$ \\
\hline$>20 \mathrm{yrs}$ & 3 & 0 & $*$ \\
\hline
\end{tabular}

* $>20$ yrs duration, the sample size was very less to correlate the findings.

Table 3: Distribution of ASB with respect to various risk factors

\begin{tabular}{|l|l|l|l|}
\hline Risk Factors & ASB + & ASB - & P Value \\
\hline AGE: & 12 & 120 & \\
$<55.36 \pm 11.02$ yrs & 20 & 98 & 0.034 \\
$>55.03 \pm 11.02$ yrs & & & \\
\hline Duration Of Diabetes & 22 & 106 & 0.017 \\
$<7.15 \pm 5.46$ yrs & 10 & 112 & 0.015 \\
$>7.15 \pm 5.46 y r s$ & 27 & 143 & 0.041 \\
\hline Glucosuria & 22 & 114 & 0.0000033 \\
\hline Ketonuria & 32 & 134 & 0.000013 \\
\hline Proteinuria & 27 & 99 & \\
\hline Pyuria & & & \\
\hline
\end{tabular}

Culture: Out of 32 diabetic cases wherein ASB was found, the most common organism responsible for ASB in diabetics was found to be E.coli (40.6\%) followed by klebsiella (31.25\%), staphylococcus aureus (25\%) and citrobacter (3.125\%).

E.coli was the main causative organism of ASB in non-diabetics as well (57.14\%). Others included klebsiella (21.43\%) and staphylococcus $(21.43 \%)$. 
The mean age of the patients with diabetes was 55.36 \pm 11.02 yrs and that of the controls was 53.12 \pm 9.31 yrs. As age increased susceptibility to ASB also increased (Table / Fig-1). Also as the duration of diabetes increased it was found that the incidence of ASB also increased (Table/Fig- 2). ASB was found to be associated with the following risk factors: $84.38 \%$ of cases with ASB had glucosuria as against $15.62 \%$ of ASB cases without glucosuria [p value=.015 and odds ratio=2.83]. Similarly $68.75 \%$ of ASB positive had ketonuria as against $31.25 \%$ of ASB cases without ketonuria [p value $=.041$ and odds ratio=1.8441]. Almost all the ASB positive cases were noted to have significant proteinuria [p value=.0000033]. 21.4\% cases with ASB had pyuria against 4\% cases with ASB but without pyuria showing a strong correlation between the two. (P value <0.05) [Table/Fig- 3]

The antimicrobials showed similar resistance pattern in both case and control groups. The resistance pattern of the organisms from the case group is illustrated in Table/fig-4.

Table 4: Antimicrobial susceptibility pattern of isolated organisms

\begin{tabular}{|l|l|l|l|l|l|l|l|l|l|l|l|l|l|}
\hline Organism & $\begin{array}{l}\text { Total } \\
\text { Samples }\end{array}$ & $\mathbf{C a c}$ & $\mathbf{C x}$ & $\mathbf{C}$ & $\mathbf{C i p}$ & $\mathbf{C d}$ & $\mathbf{C o t}$ & $\mathbf{E}$ & $\mathbf{P i t}$ & $\mathbf{L z}$ & $\mathbf{V a}$ & $\mathbf{N e t}$ & $\begin{array}{l}\text { Ge } \\
\mathbf{n}\end{array}$ \\
\hline E.coli & 13 & 10 & 13 & 13 & 9 & - & 6 & - & 13 & - & - & 11 & 12 \\
\hline Klebsiella & 10 & 9 & 10 & 10 & 6 & - & 6 & - & 10 & - & - & 10 & 10 \\
\hline Citrobacter & 1 & 1 & 0 & 1 & 0 & - & 0 & - & 1 & - & - & 1 & 1 \\
\hline Staphylococcus & 8 & - & 7 & 6 & 4 & 4 & 5 & 2 & - & 8 & 8 & 8 & 8 \\
\hline
\end{tabular}

Cac- Ceftazidime /Clavulanic acid, Cx- Cefoxitin, C- Chloramphenicol, Cip- Ciprofloxacin, Cd-Clindamycin, CotCotrimoxazole, E- Erythromycin, Pit- Piperacillin/Tazobactam, Lz-Linezolid, Va- Vancomycin, Net- Netilmicin, AkAmikacin

The organisms inoculated on UTI CHROMagar were identified within 24hrs of incubation with the following characteristic features:

E.coli - pink translucent colonies

Klebsiella - dark blue colonies

Citrobacter - magenta coloured colonies

Staphylococcus - tiny, cream- white convex colonies

\section{Discussion}

In this study we found that the frequency of asymptomatic bacteriuria is more in diabetics (12.8\%) compared to non-diabetics $(6 \%)$. There was almost 3 times increased frequency of ASB in diabetic women compared to non-diabetic women $(16 \% \mathrm{v} / \mathrm{s} 6 \%)$ but the frequency did not show much difference in male counterparts $(5.71 \% \mathrm{v} / \mathrm{s} \quad 6.09 \%)$. This was in concordance with other studies wherein in ASB was found to be higher in female diabetics $[4,16]$.

The incidence of ASB increased as the duration of diabetes increased with $p$ value $<0.05$ thus signifying its influence. E. Geerlings, Ronald P,et al also found similar results in regards to influence of duration of diabetes with respect to age[3,17]. In this study we found a significant association between glucosuria, ketonuria and ASB indicating that poorer the diabetic control greater the incidence of ASB. Proteinuria was present in all the patients with ASB indicating renal dysfunction in these individuals which may progress to further complications. $21.4 \%$ cases with ASB had pyuria against $4 \%$ cases with ASB but without pyuria showing a strong correlation between the two ( $\mathrm{P}$ value $<0.05)$. This is supported by a study done by Kelestimur F, Unal A,et al with 110 patients with diabetes, a significant association between bacteriuria , pyuria and HbA1c levels was found [18].

The most common organisms found in both case and control samples with significant bacteriuria were similar and their antimicrobial sensitivity was also similar signifying that both case and control had similar risk factors for developing antimicrobial resistance when they were enrolled in the study. Bonadio M, Mienni also proved E.coli to be the most common organism cultured from urine samples of UTI patients with 
diabetes and found that the sensitivity pattern of the organisms cultured was similar to the pattern obtained from urine samples of UTI patients without diabetes [2].

The study showed that the frequency of E.coli was higher in non-diabetics compared to diabetics [57\% v/s $40 \%$ ] which is consistent with the result of other studies conducted by Lye WC and E Geerlings $(17,19)$.

The sensitivity pattern revealed that the organisms were sensitive to most of the commonly used antibiotics with increased resistance noted to cotrimoxazole and ciprofloxacin among gram negative organisms. Staphylococcus showed some degree of resistance to ciprofloxacin, erythromycin,cotrimoxazole and clindamycin. Organisms from control group also showed similar pattern of resistance.

All the positive cultures picked up on standard medium were also picked up on UTICHROMagar and was faster and much easier as there was no need for sub-culture or other biochemical reactions for identification of organisms. Several other studies reported similar conclusion $[9,10,20]$.

Hence it was demonstrated that UTICHROMagar could be used as a primary culture medium for identification of organisms as it is easier, faster and also reliable in comparison to conventional methods as the need for subculture and other confirmatory tests is overcome.

\section{Conclusion}

we conclude that asymptomatic bacteriuria is higher is diabetic women and the risk factors increasing the incidence of ASB in diabetic patients included increased age, longer duration of diabetes, poor diabetes control indicated by glucosuria and ketonuria, macroalbuminuria and presence of pyuria. UTICHROMagar medium is an easy and faster means of identification of organisms in ASB.

\section{Funding:Nil. Conflict of interest: Nil. Permission for IRB: Yes.}

\section{References}

1. Joshi SR, Parikh RM. India--diabetes capital of the world: now heading towards hypertension. J Assoc Physicians India. 2007 May;55:323-4.
2. Asymptomatic bacteriuria in women with diabetes: Influence of metabolic control. Mario Bonadio et al, Infectious Diseases Section and metabolic unit, Department of Internal medicine, University-Hospital, Pisa, Italy

3. Geerlings SE. Urinary tract infections in patients with diabetes mellitus: epidemiology, pathogenesis and treatment. Int J Antimicrob Agents. 2008 Feb;31 Suppl 1:S54-7. Epub 2007 Dec 3.

4. Zhanel GG, Nicolle LE, Harding GK. Prevalence of asymptomatic bacteriuria and associated host factors in women with diabetes mellitus. The Manitoba Diabetic Urinary Infection Study Group, clinical infectious disease Clin Infect Dis. 1995 Aug;21(2):316-22.

5. Hansen Ro. Bacteriuria in Diabetic And NonDiabetic Out-Patients. Acta Med Scand. 1964 Dec;176:721-30.

6. Colgan R, Nicolle LE, McGlone A, Hooton TM. Asymptomatic bacteriuria in adults. Am Fam Physician. 2006 Sep 15;74(6):985-90.

7. Renko M, Tapanainen $\mathrm{P}$, Tossavainen $\mathrm{P}$, Pokka T, Uhari M. Meta-analysis of the significance of asymptomatic bacteriuria in diabetes. Diabetes Care. 2011 Jan;34(1):230-5. doi: 10.2337/dc10-0421. Epub 2010 Oct 11.

8. Lakhan Singh, Ramanesh Murthy, Hemalatha Singh, Prashanth Nigam. Asymptomatic bacteriuria in patients with type 2 diabetes mellitus. NJIRM 2013;4(6):1-4

9. D'Souza HA, Campbell M, Baron EJ. Practical bench comparison of BBL CHROMagar Orien tation and standard two-plate media for urine cultures. J Clin Microbiol. 2004 Jan;42(1):60-4.

10. Chang JC, Chien ML, Chen HM, Yan JJ, Wu JJ. Comparison of CPS ID 3 and CHROMagar Orientation chromogenic agars with standard biplate technique for culture of clinical urine samples. J Microbiol Immunol Infect. 2008 Oct;41(5):422-7.

11.Winn WC Jr, Allen S, Janda W, Koneman E, Procop G, Schreckenberger P, et al. Koneman's color atlas and textbook of diagnostic microbiology. 6th ed. Philadelphia: Lippincott Williams and Wilkins; 2006. 
12. Collee JG, Miles RS, Watt B. Tests for identification of bacteria. In: Collee JG, Fraser AG, Marmion BP, Simmons A, editors. Mackie and McCartney Practical Medical Microbiology. 14th ed, New York. Churchill Livingstone; 2006.

13. Infectious Diseases Society of America Guidelines for the Diagnosis and Treatment of Asymptomatic Bacteriuria in Adults: Lindsay E. Nicolle,Suzanne Bradley,et al.

14. Twenty-first informational Clinical and Laboratory Standards Institute. Performance standards for antimicrobial susceptibility testing. Supplement M100S21. Wayne, Pennsylvania: Clinical and Laboratory Standards Institute; 2011.

15. Isenberg, H. D. Clinical microbiology procedures handbook. Washington, D.C: American Society for Microbiology; 1992.

16. Nicolle LE. Asymptomatic bacteriuria in diabetic women. Diabetes Care. 2000 Jun;23(6):722-3.
17. Lye WC, Chan RK, Lee EJ, Kumarasinghe G. Urinary tract infections in patients with diabetes mellitus. J Infect. 1992 Mar;24(2):169-74.

18. Keleştimur F, Unal A, Paşaoğlu H, Başar E, Kiliç H, Doğanay M. [Asymptomatic bacteriuria in patients with diabetes mellitus]. Mikrobiyol Bul. 1990 Apr;24(2):126-32.

19. Geerlings SE, Stolk RP, Camps MJ, Netten PM, Hoekstra JB, Bouter KP, Bravenboer B, Collet JT, Jansz AR, Hoepelman AI. Asymptomatic bacteriuria may be considered a complication in women with diabetes. Diabetes Mellitus Women Asymptomatic Bacteriuria Utrecht Study Group. Diabetes Care. 2000 Jun;23(6):744-9.

20. Samra Z, Heifetz M, Talmor J, Bain E, Bahar J. Evaluation of use of a new chromogenic agar in detection of urinary tract pathogens. J Clin Microbiol. 1998 Apr;36(4):990-4.

\section{How to cite this article?}

Divyashree K, Yadhav K. Asymptomatic bacteriuria and associated host factors in Diabetic patients with special reference to UTI Chromagar. Int J Med Res Rev 2015;3(10):1218-1223. doi: 10.17511/ijmrr.2015.i10.221. 